\title{
On the Noisy Feedback Capacity of Gaussian Broadcast Channels
}

\author{
Sibi Raj B. Pillai \\ Department of Electrical Engineering \\ Indian Institute of Technology Bombay \\ bsraj@ee.iitb.ac.in
}

\author{
Vinod M. Prabhakaran \\ School of Technology and Computer Science \\ Tata Institute of Fundamental Research, Mumbai \\ vinodmp@tifr.res.in
}

\begin{abstract}
It is well known that, in general, feedback may enlarge the capacity region of Gaussian broadcast channels. This has been demonstrated even when the feedback is noisy (or partial-but-perfect) and only from one of the receivers. The only case known where feedback has been shown not to enlarge the capacity region is when the channel is physically degraded. In this paper, we show that for a class of two-user Gaussian broadcast channels (not necessarily physically degraded), passively feeding back the stronger user's signal over a link corrupted by Gaussian noise does not enlarge the capacity region if the variance of feedback noise is above a certain threshold.
\end{abstract}

\section{INTRODUCTION}

It is well known that feedback does not increase the capacity of a memoryless point-to-point channel, a result which goes back to C. E. Shannon [1]. However, feedback has a positive impact in simplifying coding schemes and boosting error exponents [2]. With the discovery of capacity regions for several multiuser models in the ' 70 s and ' 80 s, it was of interest to find the impact of feedback in these models. For the discrete memoryless broadcast channel (BC), El Gamal [3] showed that feedback does not enlarge the capacity region when the channel is physically degraded, and Dueck [4] demonstrated a broadcast channel for which points outside its capacity region can be attained using feedback.

For a two-user scalar Gaussian broadcast channel (GBC), El Gamal [5] showed that the capacity region is unchanged by the presence of noiseless feedback, if one of the receivers is physically degraded with respect to the other. However, Ozarow and Leung [6] showed the surprising fact that the GBC capacity region is enlarged by feedback for a class of positively correlated noise processes at the receivers. The technique of [6] used full causal feedback from both the receivers. Recently it was shown that even perfect causal feedback from one of the receivers can enlarge the GBC capacity region [7]. In other related works, it was shown that the capacity enlargement can occur for the discrete memoryless broadcast channel even when the feedback is noisy [8], [9], or rate-limited [10], [11]. The GBC case was also considered in [9] and [11]. For the Gaussian multiple-access channel (MAC), it is known that noisy feedback, even to only one of the transmitters, always enlarges the capacity region [12]. Moreover, a duality has been shown between linear coding schemes for MAC and $\mathrm{BC}$ in the presence of noiseless feedback [13]. Thus, in general, there is an optimism about the availability of feedback enlarging the capacity region of broadcast channels when the channel is not physically degraded.

Against this backdrop, the purpose of this paper is to show that the anticipated capacity enlargement may not exist for all feedback models over a GBC. We show that, for a class of two-user scalar GBCs, when the stronger receiver's signal (i.e., the signal of the receiver with the smaller noise variance) is passively fed back to the transmitter over a noisy link corrupted by independent Gaussian noise, any capacity enlargement is impossible if the feedback noise variance is above a certain threshold. Our class of channels is, in fact, the same as that studied by Ozarow and Leung [6] where they showed that the capacity region is enlarged by perfect feedback from both receivers. This class includes independent noises at the two users as well as a range of positive correlations.

We also study a related class of vector GBCs with partialbut-perfect feedback. Specifically, consider a vector GBC with a strong receiver employing two receive antennas and the other receiver and encoder only having single antennas. In the absence of feedback, the optimal scheme is superposition coding, along with maximal ratio combining at the strong receiver. Now, assume that the weak user has a higher noise variance with respect to the first antenna of the strong receiver. Then, we will show that even perfectly feeding back the second antenna output from the strong receiver does not enlarge the capacity region.

The organization of this paper is as follows. In the next section, we will describe the broadcast channel and the feedback model. This will be followed by our main result in Section III, which shows that feedback from the stronger user does not enlarge the capacity region if the feedback noise variance is above a certain threshold for a class of Gaussian noise models. A Gaussian BC with two antennas at the strong receiver and perfect feedback from one of the antennas will be considered in Section IV. Section V concludes the paper.

\section{SySTEM Model}

Consider a memoryless two-user scalar Gaussian broadcast channel, where receiver 1 is the stronger receiver (i.e., its noise variance is not larger than the noise variance of the other user). Assume a noisy feedback link from the strong receiver to the transmitter, as shown in Figure 1. 


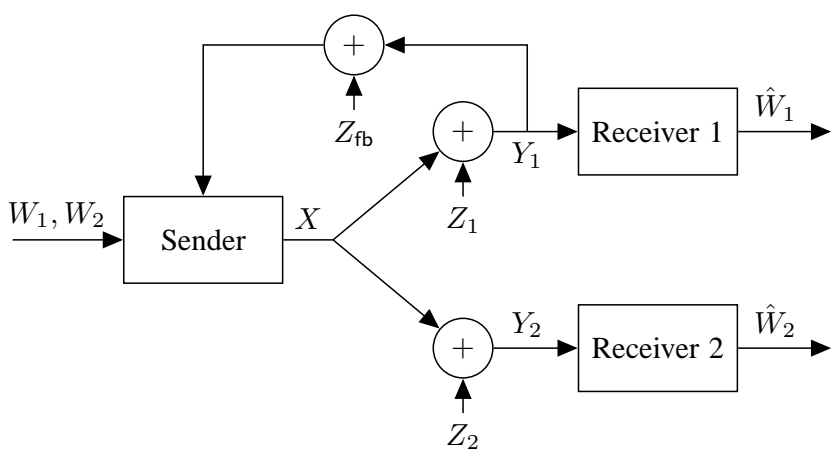

Fig. 1. Scalar Gaussian broadcast channel with noisy feedback (causal)

In this model, $X$ represents the transmitted signal. The additive (forward) channel noises $Z_{1}, Z_{2}$ are zero-mean jointly Gaussian with variances $\sigma_{1}^{2}, \sigma_{2}^{2}$, respectively, and correlation coefficient $\rho$. As mentioned above, we will take $\sigma_{1}^{2} \leq \sigma_{2}^{2}$. The additive noise on the feedback channel $Z_{\mathrm{fb}}$ is assumed to be independent of $\left(Z_{1}, Z_{2}\right)$ and zero-mean Gaussian with variance $\sigma_{\mathrm{fb}}^{2}$. In this setup, we aim to send two independent messages, say $W_{1}$ and $W_{2}$, to the respective receivers. The transmitted symbol at the $i^{\text {th }}$ instant can be a function of the messages and the causal but noisy feedback of the stronger user's signal, i.e.,

$$
X_{i}=g_{i}\left(W_{1}, W_{2}, Y_{1}^{i-1}+Z_{\mathrm{fb}}^{i-1}\right),
$$

where the symbol $U^{i-1} \triangleq\left(U_{1}, \cdots, U_{i-1}\right)$. We will convey a pair of messages in $n$ uses of the channel, and the alphabet sizes of $W_{1}, W_{2}$ are $2^{n R_{1}}$ and $2^{n R_{2}}$, respectively. Consider the average probability of error over a uniform choice of the messages, which we denote by $P_{e}(n)$. We are interested in the capacity region $\mathcal{C}_{\mathrm{sbc}}^{\text {noisy }} \mathrm{fb}$ of our model under an average transmit power constraint of $P$.

Definition 1. The capacity region $\mathcal{C}_{\mathrm{sbc}}^{\text {noisy-fb }}$ is the closure of the set of all rate-pairs $\left(R_{1}, R_{2}\right)$ such that there is a sequence of encoder-decoders with $P_{e}(n) \rightarrow 0$ as $n \rightarrow \infty$.

Let $\mathcal{C}_{\mathrm{sbc}}^{\text {wo }}$ fb denote the capacity region without feedback. Clearly,

$$
\mathcal{C}_{\text {sbc }}^{\text {wo }}{ }^{\mathrm{fb}} \subseteq \mathcal{C}_{\mathrm{sbc}}^{\text {noisy }-\mathrm{fb}}
$$

The region $\mathcal{C}_{\mathrm{sbc}}^{\text {wo }-\mathrm{fb}}$ is a well known quantity, which can be achieved by superposition coding [14].

\section{Feedback Noise Variance Thresholds}

Our main result establishes a threshold for the feedback noise variance beyond which the feedback from stronger user does not enlarge the capacity region for a class of forward noise correlations (including independent and physically degraded cases).

Theorem 1. For $0 \leq \rho \leq \sqrt{\frac{\sigma_{1}^{2}}{\sigma_{2}^{2}}} \leq 1, \mathcal{C}_{\mathrm{sbc}}^{\text {noisy }-\mathrm{fb}}=\mathcal{C}_{\mathrm{sbc}}^{\text {wo }-\mathrm{fb}}$

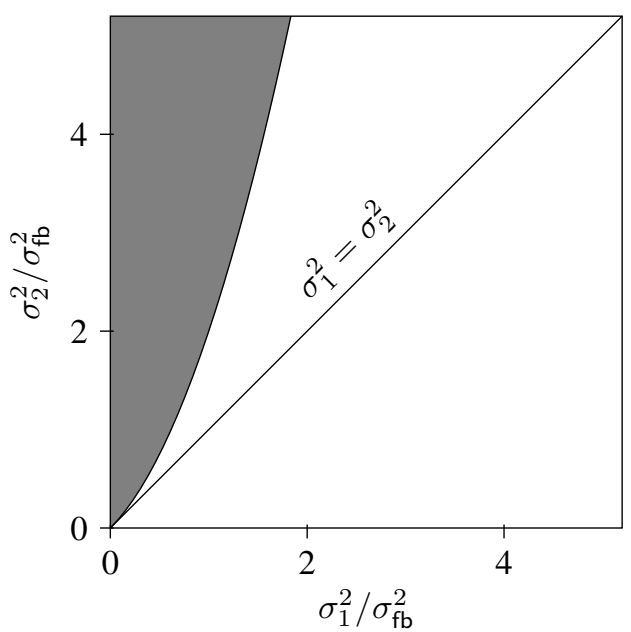

Fig. 2. Theorem 1 guarantees that for independent forward channel noises, noisy feedback from the stronger user does not enlarge the capacity region when the noise variances fall in the shaded region.

\section{whenever}

$$
\frac{\sigma_{\mathrm{fb}}^{2}}{\sigma_{1}^{2}} \geq \frac{1}{\frac{\sigma_{2}^{2}}{\sigma_{1}^{2}}-1}\left(1-\rho \sqrt{\frac{\sigma_{2}^{2}}{\sigma_{1}^{2}}}\right)^{2} .
$$

Notice that, when $\rho=\sqrt{\frac{\sigma_{1}^{2}}{\sigma_{2}^{2}}}$, the broadcast channel is physically degraded and the feedback noise variance threshold in (2) is 0 . This is already implied by El Gamal's result [5]. At the other extreme, when the forward channel noises are independent $(\rho=0)$, the result is as shown in Fig. 2 . However, note that the theorem does not give a threshold for all positively correlated $Z_{1}, Z_{2}$. For example, when $Z_{2}=2 Z_{1}$, we have $1=\rho \geq \sqrt{\frac{\sigma_{1}^{2}}{\sigma_{2}^{2}}}=\frac{1}{2}$, and Theorem 1 does not apply. More specifically, the theorem above gives a threshold whenever we can write $Z_{1}, Z_{2}$ as (3)-(4), where $Z, \tilde{Z}_{1}, \tilde{Z}_{2}$ are independent. This is also the class of channels studied by Ozarow and Leung [6].

Proof: We start by noting that when $\sigma_{1}^{2} \leq \sigma_{2}^{2}$ and $\rho \leq$ $\sqrt{\frac{\sigma_{1}^{2}}{\sigma_{2}^{2}}}$, without loss of generality, we may write $Z_{1}, Z_{2}$ in the
form

$$
\begin{aligned}
& Z_{1}=Z+\tilde{Z}_{1} \\
& Z_{2}=Z+\tilde{Z}_{2},
\end{aligned}
$$

where $Z, \tilde{Z}_{1}, \tilde{Z}_{2}$ are independent zero-mean Gaussian random variables with variances $\varsigma^{2}, \varsigma_{1}^{2}, \varsigma_{2}^{2}$, respectively, given by

$$
\begin{aligned}
& \varsigma^{2}=\rho \sqrt{\sigma_{1}^{2} \sigma_{2}^{2}} \\
& \varsigma_{1}^{2}=\sigma_{1}^{2}-\rho \sqrt{\sigma_{1}^{2} \sigma_{2}^{2}} \\
& \varsigma_{2}^{2}=\sigma_{2}^{2}-\rho \sqrt{\sigma_{1}^{2} \sigma_{2}^{2}} .
\end{aligned}
$$

The key idea of the proof is to give receiver 1 access to the feedback noise (step (a) below). By Fano's inequality, 
(suppressing an $n \epsilon$ term)

$$
\begin{aligned}
n R_{1} & \approx I\left(W_{1} ; Y_{1}^{n} \mid W_{2}\right) \\
& \stackrel{\text { (a) }}{\leq} I\left(W_{1} ; Y_{1}^{n}, Z_{\mathrm{fb}}^{n} \mid W_{2}\right) \\
& =\sum_{i=1}^{n} I\left(W_{1} ; Y_{1 i} \mid W_{2}, Y_{1}^{i-1}, Z_{\mathrm{fb}}^{i-1}\right) \\
& =\sum_{i=1}^{n} h\left(Y_{1 i} \mid W_{2}, Y_{1}^{i-1}, Z_{\mathrm{fb}}^{i-1}\right)-h\left(Y_{1 i} \mid W_{1}, W_{2}, Y_{1}^{i-1}, Z_{\mathrm{fb}}^{i-1}\right) \\
& \stackrel{\text { (b) }}{\leq} \sum_{i=1}^{n} h\left(Y_{1 i} \mid W_{2}, Y_{1}^{i-1}-\alpha Z_{\mathrm{fb}}^{i-1}\right)-h\left(Z_{1 i}\right) \\
& =\sum_{i=1}^{n} h\left(Y_{1 i} \mid W_{2}, X^{i-1}+Z^{i-1}+\tilde{Z}_{1}^{i-1}-\alpha Z_{\mathrm{fb}}^{i-1}\right)-h\left(Z_{1 i}\right),
\end{aligned}
$$

where in (b) we used the fact that $X_{i}$ is a function of $\left(W_{1}, W_{2}, Y_{1}^{i-1}, Z_{\mathrm{fb}}^{i-1}\right)$. We choose $\alpha=\frac{\varsigma_{1}^{2}}{\sigma_{\mathrm{fb}}^{2}}$ so that $\tilde{Z}_{1}-\alpha Z_{\mathrm{fb}}$ is independent of $\tilde{Z}_{1}+Z_{\mathrm{fb}}$. This choice ensures that $\tilde{Z}_{1}-\alpha Z_{\mathrm{fb}}$ is independent of $Z_{1}+Z_{\mathrm{fb}}=Z+\tilde{Z}_{1}+Z_{\mathrm{fb}}$. Notice that (i) $X_{i}$ is a function only of everything the encoder knows before time instant $i$, namely, $\left(W_{1}, W_{2}, Z_{1}^{i-1}+Z_{\mathrm{fb}}^{i-1}\right)$, and (ii) both $\tilde{Z}_{1}^{i-1}-\alpha Z_{\mathrm{fb}}^{i-1}$ and $\tilde{Z}_{2}^{i-1}$ are independent of encoder's knowledge $\left(W_{1}, W_{2}, Z_{1}^{i-1}+Z_{\mathrm{fb}}^{i-1}\right)$ before time instant $i$. Now, if $\operatorname{Var}\left(\tilde{Z}_{1}-\alpha Z_{\mathrm{fb}}\right) \leq \operatorname{Var}\left(\tilde{Z}_{2}\right)$, for each $i$, then we can write ${ }^{1}$

$$
\begin{aligned}
& h\left(Y_{1 i} \mid W_{2}, X^{i-1}+Z^{i-1}+\tilde{Z}_{1}^{i-1}-\alpha Z_{\mathrm{fb}}^{i-1}\right) \\
& \quad \leq h\left(Y_{1 i} \mid W_{2}, X^{i-1}+Z^{i-1}+\tilde{Z}_{2}^{i-1}\right) .
\end{aligned}
$$

It is easy to verify that the condition $\operatorname{Var}\left(\tilde{Z}_{1}-\alpha Z_{\mathrm{fb}}\right) \leq$ $\operatorname{Var}\left(\tilde{Z}_{2}\right)$ is precisely (2). Substituting back, we have

$$
n R_{1} \leq\left(\sum_{i=1}^{n} h\left(Y_{1 i} \mid W_{2}, Y_{2}^{i-1}\right)\right)-\frac{n}{2} \log 2 \pi e \sigma_{1}^{2} .
$$

By Fano's inequality,

$$
\begin{aligned}
n R_{2} & \approx I\left(W_{2} ; Y_{2}^{n}\right) \\
& =h\left(Y_{2}^{n}\right)-h\left(Y_{2}^{n} \mid W_{2}\right) \\
& \leq \sum_{i=1}^{n} h\left(Y_{2 i}\right)-h\left(Y_{2}^{n} \mid W_{2}\right)
\end{aligned}
$$

${ }^{1}$ To see this, let us write $\tilde{Z}_{2}=\tilde{Z}_{2 a}+\tilde{Z}_{2 b}$, where $\tilde{Z}_{2 a}, \tilde{Z}_{2 b}$ are independent and also independent of everything else. Let $\operatorname{Var}\left(\tilde{Z}_{2 a}\right)=\operatorname{Var}\left(\tilde{Z}_{1}-\alpha Z_{\mathrm{fb}}\right)$. Then, we may write

$$
\begin{aligned}
h\left(Y_{1 i} \mid W_{2}, X^{i-1}+Z^{i-1}+\right. & \left.\tilde{Z}_{1}^{i-1}-\alpha Z_{\mathrm{fb}}^{i-1}\right) \\
& \leq h\left(Y_{1 i} \mid W_{2}, X^{i-1}+Z^{i-1}+\tilde{Z}_{2 a}^{i-1}\right),
\end{aligned}
$$

since the independence of $\left(\tilde{Z}_{1}^{i-1}-\alpha Z_{\mathrm{fb}}^{i-1}\right)$ and everything the encoder has access to before time instant $i$ allows us to replace $\tilde{Z}_{1}^{i-1}-\alpha Z_{\mathrm{fb}}^{i-1}$ by any other memoryless Gaussian random vector $\left(\tilde{Z}_{2 a}^{i-1}\right.$ in our case) with the same variance and which is also independent of encoder's knowledge before time $i$. Now, using the fact that $Z_{2 b}^{i-1}$ is independent of everything else, we have

$$
\begin{aligned}
h\left(Y_{1 i} \mid W_{2}, X^{i-1}+Z^{i-1}\right. & \left.+\tilde{Z}_{2 a}^{i-1}\right) \\
& =h\left(Y_{1 i} \mid W_{2}, X^{i-1}+Z^{i-1}+\tilde{Z}_{2 a}^{i-1}, \tilde{Z}_{2 b}^{i-1}\right) \\
& \leq h\left(Y_{1 i} \mid W_{2}, X^{i-1}+Z^{i-1}+\tilde{Z}_{2 a}^{i-1}+\tilde{Z}_{2 b}^{i-1}\right) .
\end{aligned}
$$

$$
\leq \frac{n}{2} \log 2 \pi e\left(P+\sigma_{2}^{2}\right)-h\left(Y_{2}^{n} \mid W_{2}\right) .
$$

where the last inequality follows (via concavity of log) from the power constraint and the memorylessness of the channel. We can relate the first term of (5) and the second term above through the entropy power inequality (EPI).

\section{Lemma 2.}

$$
2^{\frac{2}{n} h\left(Y_{2}^{n} \mid W_{2}\right)} \geq 2^{\frac{2}{n} \sum_{i=1}^{n} h\left(Y_{1 i} \mid W_{2}, Y_{2}^{i-1}\right)}+2 \pi e\left(\sigma_{2}^{2}-\sigma_{1}^{2}\right) .
$$

The proof, which is along the lines of El Gamal [5, Lemma 1] and Blachman [15], is given in the appendix.

To finish the proof, we note that

$$
\frac{n}{2} \log 2 \pi e\left(P+\sigma_{2}^{2}\right) \geq h\left(Y_{2}^{n}\right) \geq h\left(Y_{2}^{n} \mid W_{2}\right) \geq h\left(Z_{2}^{n}\right),
$$

where the last inequality follows from $h\left(Y_{2}^{n} \mid W_{2}\right)=$ $\sum_{i=1}^{n} h\left(Y_{2 i} \mid W_{2}, Y_{2}^{i-1}\right) \leq \sum_{i=1}^{n} h\left(Y_{2 i} \mid X_{i}, W_{2}, Y_{2}^{i-1}\right)=$ $h\left(Z_{2}^{n}\right)$. Hence, there is some $\theta \in[0,1]$ such that

$$
\frac{1}{n} h\left(Y_{2}^{n} \mid W_{2}\right)=\frac{1}{2} \log 2 \pi e\left(\theta P+\sigma_{2}^{2}\right) .
$$

Substituting in (6), we get

$$
R_{2} \leq \frac{1}{2} \log \left(1+\frac{(1-\theta) P}{\theta P+\sigma_{2}^{2}}\right) .
$$

Furthermore, (5) and (7) will imply,

$$
R_{1} \leq \frac{1}{2} \log \left(1+\frac{\theta P}{\sigma_{1}^{2}}\right) .
$$

Thus, we have shown that $\left(R_{1}, R_{2}\right) \in \mathcal{C}_{\mathrm{sbc}}^{\text {wo-fb }}$, and the proof is complete.

Theorem 1 can also be extended to more generalized feedback settings with additive Gaussian noise where the feedback noise may not be independent of the forward channel noises. This extension will be considered in a longer version of this paper.

\section{Vector Broadcast Channel with FeEdback}

It may appear that the additive Gaussian noise in the feedback plays a critical role in the negative result that we presented in the last section. In this section we study a vector GBC where the signal from one of the antennas will be fed back perfectly, but this still does not enlarge the capacity region in certain settings as we will describe below.

Let us consider the two-user memoryless broadcast channel shown in Figure 3 below, where user 1 makes two independent observations $\left(Y_{11}, Y_{12}\right)$ of each transmitted symbol. User 2 observes a scalar output $Y_{2}$. For simplicity, we will assume that the noises on different antennas are independent. Specifically, let $Z=\left(Z_{2}, Z_{11}, Z_{12}\right)$ be a zero mean Gaussian random vector with a diagonal covariance matrix

$$
K_{z}=\left[\begin{array}{ccc}
\sigma_{2}^{2} & 0 & 0 \\
0 & \sigma_{11}^{2} & 0 \\
0 & 0 & \sigma_{12}^{2}
\end{array}\right]
$$

Let us assume that the output symbols $Y_{12}$ are perfectly fed back to the transmitter (causally). Notice that $Y_{11}$ as well as $Y_{2}$ 


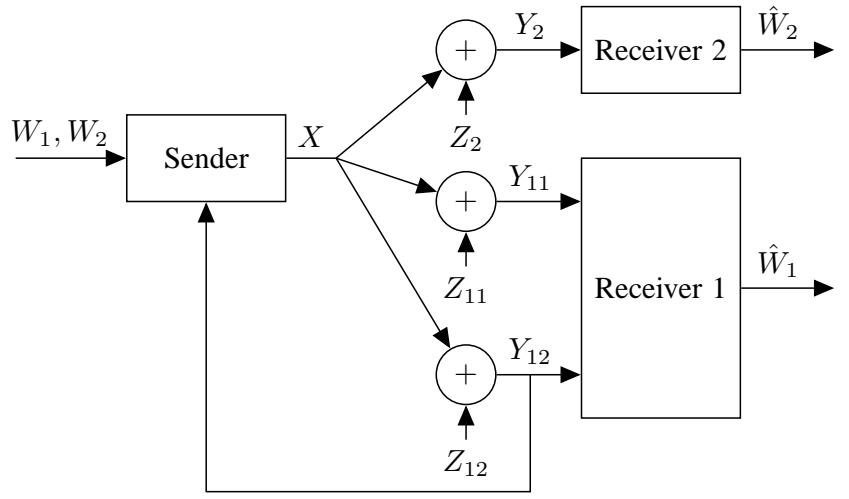

Fig. 3. A vector Gaussian broadcast channel with feedback

are not fed back. Let $\mathcal{C}_{\mathrm{vbc}}^{\text {partial-fb }}$ and $\mathcal{C}_{\mathrm{vbc}}^{\mathrm{wo}-\mathrm{fb}}$ denote the feedback and no-feedback capacity regions.

We study the case where $\sigma_{11}^{2} \leq \sigma_{2}^{2}$, i.e., the second receiver has a larger noise variance than the noise variance at the antenna of the first receiver that is not fed back. Under this assumption, we will show that perfect causal feedback of $Y_{12}$ does not enlarge the capacity region. Notice that the 'no enlargement' result holds good irrespective of the link quality of $Y_{12}$. This is surprising, since we are feeding back a major portion of the output to receiver 1, particularly so when $\sigma_{12}^{2}<\sigma_{11}^{2}$. We now state our main theorem.

Theorem 3. $\mathcal{C}_{\mathrm{vbc}}^{\text {partial-fb }}=\mathcal{C}_{\mathrm{vbc}}^{\text {wo-fb }}$ if $\sigma_{11}^{2} \leq \sigma_{2}^{2}$.

The proof is along the same lines as that of Theorem 1 presented in the last section.

Proof: Recall that the boundary of the no-feedback capacity region $\mathcal{C}_{\mathrm{vbc}}^{\mathrm{wo}-\mathrm{fb}}$ is given by

$$
\begin{aligned}
& R_{1} \leq \frac{1}{2} \log \left(1+\theta P\left[\frac{1}{\sigma_{11}^{2}}+\frac{1}{\sigma_{12}^{2}}\right]\right), \\
& R_{2} \leq \frac{1}{2} \log \left(1+\frac{(1-\theta) P}{\theta P+\sigma_{2}^{2}}\right),
\end{aligned}
$$

for $\theta \in[0,1]$. This is just the capacity region of the scalar broadcast channel resulting from receiver 1 pre-processing $Y_{11}$ and $Y_{12}$ by passing it through a maximal ratio combiner.

By Fano's inequality,

$$
\begin{aligned}
n R_{2} & \approx I\left(W_{2} ; Y_{2}^{n}\right) \\
& =h\left(Y_{2}^{n}\right)-h\left(Y_{2}^{n} \mid W_{2}\right) \\
& \leq \frac{n}{2} \log \pi e\left(P+\sigma_{2}^{2}\right)-h\left(Y_{2}^{n} \mid W_{2}\right) .
\end{aligned}
$$

We know that there exists some $\theta \in[0,1]$ such that

$$
h\left(Y_{2}^{n} \mid W_{2}\right)=\frac{n}{2} \log 2 \pi e\left(\theta P+\sigma_{2}^{2}\right) .
$$

Thus,

$$
R_{2} \leq \frac{1}{2} \log \left(1+\frac{(1-\theta) P}{\theta P+\sigma_{2}^{2}}\right)
$$

Let us now consider the rates for receiver 1 . We will write $\left(Y_{11}, Y_{12}\right)$ as $\mathbf{Y}_{1}$, and let $\mathbf{Y}_{1}^{n}$ denote the $2 \times n$ matrix of received values at receiver 1. Again, by Fano's inequality

$$
\begin{aligned}
n R_{1} & \approx I\left(W_{1} ; \mathbf{Y}_{1}^{n} \mid W_{2}\right) \\
& =\sum_{i=1}^{n} I\left(W_{1} ; \mathbf{Y}_{1, i} \mid W_{2}, \mathbf{Y}_{1}^{i-1}\right) .
\end{aligned}
$$

Let $\frac{1}{\sigma_{e}^{2}}=\frac{1}{\sigma_{11}^{2}}+\frac{1}{\sigma_{12}^{2}}$ and consider the following invertible transformation of $\mathbf{Y}_{1}^{12}$.

$$
\left[\begin{array}{c}
Y_{e} \\
Y_{e}^{\perp}
\end{array}\right]=\sigma_{e}^{2}\left[\begin{array}{cc}
\frac{1}{\sigma_{11}^{2}} & \frac{1}{\sigma_{12}^{2}} \\
-1 & +1
\end{array}\right] \mathbf{Y}=\left[\begin{array}{c}
X+Z_{e} \\
Z_{e}^{\perp}
\end{array}\right]
$$

where $Z_{e}=\sigma_{e}^{2}\left(\frac{Z_{11}}{\sigma_{11}^{2}}+\frac{Z_{12}}{\sigma_{12}^{2}}\right)$ is independent of $Z_{e}^{\perp}$. Note that $Z_{e} \sim \mathcal{N}\left(0, \sigma_{e}^{2}\right)$ is an independent identically distributed (i.i.d.) process with $\mathbb{E}\left[Z_{e} Z_{12}^{*}\right]=\frac{\sigma_{11}^{2} \sigma_{12}^{2}}{\sigma_{11}^{2}+\sigma_{12}^{2}}$. Since the $Z_{e}^{\perp}$ process is i.i.d. and independent of the $Z_{e}$ process, we can write,

$$
\begin{aligned}
\sum_{i=1}^{n} I\left(W_{1} ;\right. & \left.\mathbf{Y}_{1, i} \mid W_{2}, \mathbf{Y}_{1}^{i-1}\right) \\
& =\sum_{i=1}^{n} h\left(Y_{e, i} \mid W_{2}, \mathbf{Y}_{1}^{i-1}\right)-h\left(Z_{e, i}\right) \\
& \stackrel{(a)}{\leq} \sum_{i=1}^{n} h\left(Y_{e, i} \mid W_{2}, Y_{11}^{i-1}\right)-h\left(Z_{e, i}\right) \\
& =\sum_{i=1}^{n} h\left(X+Z_{e, i} \mid W_{2}, Y_{11}^{i-1}\right)-h\left(Z_{e, i}\right),
\end{aligned}
$$

where in step (a) we removed $Y_{12}^{i-1}$ from the conditioning to obtain the inequality. Since $Y_{2}$ has a larger noise variance than $Y_{11}$ (i.e., $\sigma_{2}^{2} \geq \sigma_{11}^{2}$ ) and both of them are not fed back to the transmitter, we may write (also see footnote 1)

$$
n R_{1} \leq \sum_{i=1}^{n} h\left(X_{i}+Z_{e, i} \mid Y_{2}^{i-1}, W_{2}\right)-h\left(Z_{e, i}\right) .
$$

Consider an i.i.d. process $Z_{d} \sim \mathcal{N}\left(0, \sigma_{2}^{2}-\sigma_{e}^{2}\right)$, independent of all other processes mentioned before. Using El Gamal's version of EPI [5, Lemma 1],

$$
\begin{aligned}
2^{\frac{2}{n} \sum_{i=1}^{n} h\left(Y_{e, i} \mid Y_{2}^{i-1}, W_{2}\right)} & \leq 2^{\frac{2}{n} h\left(Y_{e}^{n}+Z_{d}^{n} \mid W_{2}\right)}-2 \pi e\left(\sigma_{2}^{2}-\sigma_{e}^{2}\right) \\
& =2^{\frac{2}{n} h\left(Y_{2}^{n} \mid W_{2}\right)}-2 \pi e\left(\sigma_{2}^{2}-\sigma_{e}^{2}\right) \\
& =2 \pi e\left(\theta P+\sigma_{2}^{2}\right)-2 \pi e\left(\sigma_{2}^{2}-\sigma_{e}^{2}\right) \\
& =2 \pi e\left(\theta P+\sigma_{e}^{2}\right) .
\end{aligned}
$$

Collecting all these together, we have

$$
R_{1} \leq \frac{1}{2} \log \left(1+\frac{\theta P}{\sigma_{e}^{2}}\right) .
$$

This completes our converse, and we have shown whenever $\sigma_{11}^{2} \leq \sigma_{2}^{2}$, perfect causal feedback from the antenna $Y_{12}$ does not change the capacity region of our broadcast channel.

While we have assumed independent noise processes at the antennas, this can be extended to the case where the noises may be correlated. In fact, Theorem 1 can be obtained as a corollary of such a generalization. 


\section{CONCLUSiON}

We presented a class of two-user scalar Gaussian broadcast channels with passive noisy feedback from one of the receivers for which feedback does not enlarge the capacity region. Our result is in the form of a threshold on the feedback noise variance above which feedback cannot achieve points outside the no-feedback capacity region. We also saw a class of two-user vector Gaussian broadcast channels where perfect feedback of some components of the received signal from a user does not lead to an enlarged capacity region.

Our study raises the question whether the threshold in Theorem 1 is tight, i.e., is there a coding scheme which can achieve points outside the no-feedback capacity region whenever the feedback noise variance is below our threshold (and also in all cases where Theorem 1 does not apply).

It must be emphasized that we only considered passive feedback. With active feedback schemes no such thresholds may exist, e.g., one simple way to get around the limitation is to conserve power on the feedback link and use it only every so often (or equivalently, to send only a subsampled version of the received signal, but repeat each sample several times) so that the effective noise variance on the feedback link is below our threshold. This suggests that it is unlikely that there are any such thresholds for active feedback schemes with a noisy link. The same comment applies to rate-limited feedback [11].

\section{ACKNOWLEDGEMENTS}

This research was supported in part by Information Technology Research Academy (ITRA), Government of India under ITRA-Mobile grant ITRA/15(64)/Mobile/USEAADWN/01. SRBP's research was also supported by the Department of Science \& Technology (DST) India grant SB/S3/EECE/077/2013. VP's research was partially supported by a Ramanujan Fellowship from the Department of Science \& Technology, Government of India.

\section{REFERENCES}

[1] C. E. Shannon, "The zero-error capacity of a noisy channel," IRE Transactions on Information Theory, vol. 19, 1956.

[2] A. El Gamal and Y.-H. Kim, Network Information Theory. Cambridge University Press, 2011.

[3] A. El Gamal, "The feedback capacity of degraded broadcast channels," IEEE Transactions on Information Theory, vol. IT-24, pp. 379-381, 1978.

[4] G. Dueck, "Partial feedback for two-way and broadcast channels," Information and Control, vol. 46, no. 1, pp. 1-15, 1980.

[5] A. El Gamal, "The capacity of the physically degraded Gaussian broadcast channel with feedback (corresp.)," IEEE Transactions on Information Theory, vol. 27, no. 4, pp. 508-511, Jul 1981.

[6] L. H. Ozarow and S. K. Leung-Yan-Cheong, "An achievable region and outer bound for the Gaussian broadcast channel with feedback," IEEE Transactions on Information Theory, vol. IT-30, pp. 667-671, 1984.

[7] S. R. Bhaskaran, "Gaussian BC with feedback," IEEE Transactions on Information Theory, pp. 5252-5257, Aug 2008.

[8] O. Shayevitz and M. Wigger, "On the capacity of the discrete memoryless broadcast channel with feedback," IEEE Transactions on Information Theory, vol. 59, no. 3, pp. 1329-1345, Mar 2013.

[9] R. Venkataramanan and S. Pradhan, "An achievable rate region for the broadcast channel with feedback," IEEE Transactions on Information Theory, vol. 59, no. 10, pp. 6175-6191, Oct 2013.
[10] Y. Wu and M. Wigger, "Any positive feedback rate increases the capacity of strictly less-noisy broadcast channels," in Proc. of Information Theory Workshop (ITW) 2013, pp. 1-5, Sept 2013.

[11] _ _ "Coding schemes for discrete memoryless broadcast channels with rate-limited feedback," CoRR, vol. abs/1401.6219, 2014.

[12] A. Lapidoth and M. Wigger, "On the AWGN MAC with imperfect feedback," IEEE Transactions on Information Theory, vol. 56, no. 11, pp. 5432-5476, Nov 2010.

[13] B. Amor, Y. Steinberg, and M. Wigger, "MIMO MAC-BC duality with linear-feedback coding schemes," CoRR, vol. abs/1404.2584, 2014.

[14] T. M. Cover and J. A. Thomas, Elements of Information Theory. Wiley, 1991.

[15] N. M. Blachman, "The convolution inequality for entropy powers," IEEE Transactions on Information Theory, vol. 11, no. 2, pp. 267-271, Apr 1965.

\section{APPENDIX}

Proof of Lemma 2:

We prove this by induction on $n$. For $n=1$, the inequality follows from entropy power inequality [2, pg. 22] since we may write

$$
h\left(Y_{2,1} \mid W_{2}\right)=h\left(Y_{1,1}+Z_{2,1}^{\prime} \mid W_{2}\right),
$$

where $Z_{2,1}^{\prime}$ is zero-mean Gaussian with

$$
\operatorname{Var}\left(Z_{2,1}^{\prime}\right)=\sigma_{2}^{2}-\sigma_{1}^{2}
$$

and independent of everything else. This simply follows from the fact that $\left(Z_{1,1}, Z_{2,1}\right)$ is independent of $\left(W_{2}, X_{1}\right)$, and $\operatorname{Var}\left(Z_{1,1}\right)=\sigma_{1}^{2} \leq \sigma_{2}^{2}=\operatorname{Var}\left(Z_{2,1}\right)$.

Suppose (7) is true for $n=m-1$. We may write

$$
h\left(Y_{2 m} \mid\left(W_{2}, Y_{2}^{m-1}\right)\right)=h\left(Y_{1 m}+Z_{2 m}^{\prime} \mid\left(W_{2}, Y_{2}^{m-1}\right)\right),
$$

where $Z_{2 m}^{\prime}$ is zero-mean Gaussian with

$$
\operatorname{Var}\left(Z_{2 m}^{\prime}\right)=\sigma_{2}^{2}-\sigma_{1}^{2}
$$

and independent of everything else. This follows from the fact that $\left(Z_{1 m}, Z_{2 m}\right)$ is independent of $\left(W_{2}, Y_{2}^{m-1}, X_{m}\right)$ and $\operatorname{Var}\left(Z_{1 m}\right) \leq \operatorname{Var}\left(Z_{2 m}\right)$. By (conditional) entropy power inequality [2, pg. 22],

$$
2^{2 h\left(Y_{2 m} \mid W_{2}, Y_{2}^{m-1}\right)} \geq 2^{2 h\left(Y_{1 m} \mid W_{2}, Y_{2}^{m-1}\right)}+2^{2 h\left(Z_{2}^{\prime}\right)} .
$$

i.e.,

$2 h\left(Y_{2 m} \mid W_{2}, Y_{2}^{m-1}\right) \geq \log \left(2^{2 h\left(Y_{1 m} \mid W_{2}, Y_{2}^{m-1}\right)}+2 \pi e\left(\sigma_{2}^{2}-\sigma_{1}^{2}\right)\right)$.

So,

$$
\begin{aligned}
& \frac{2}{m} h\left(Y_{2}^{m} \mid W_{2}\right) \\
& =\frac{m-1}{m} \frac{2}{m-1} h\left(Y_{2}^{m-1} \mid W_{2}\right)+\frac{2}{m} h\left(Y_{2 m} \mid W_{2}, Y_{2}^{m-1}\right) \\
& \stackrel{\text { (a) }}{\geq} \frac{m-1}{m} \log \left(2^{\frac{2}{m-1} \sum_{i=1}^{m-1} h\left(Y_{1 i} \mid W_{2}, Y_{2}^{i-1}\right)}+2 \pi e\left(\sigma_{2}^{2}-\sigma_{1}^{2}\right)\right) \\
& +\frac{1}{m} \log \left(2^{2 h\left(Y_{1 m} \mid W_{2}, Y_{2}^{m-1}\right)}+2 \pi e\left(\sigma_{2}^{2}-\sigma_{1}^{2}\right)\right) \\
& \stackrel{\text { (b) }}{\geq} \log \left(2^{\frac{2}{m} \sum_{i=1}^{m} h\left(Y_{1 i} \mid W_{2}, Y_{2}^{i-1}\right)}+2 \pi e\left(\sigma_{2}^{2}-\sigma_{1}^{2}\right)\right) \text {. }
\end{aligned}
$$

where (a) follows from the induction hypothesis and the EPI above, and (b) follows from convexity of $\log \left(2^{u}+v\right)$ in $u$ for $v \geq 0$. 